\title{
A practical approach to the patient with chronic diarrhoea
}

\author{
Authors: George $E$ Hiner $^{A}$ and Julian RF Walters ${ }^{B}$
}

Chronic diarrhoea is common, occurring as the first presentation of several diagnoses, or as a prolonged disorder where stool frequency, urgency and incontinence have major impacts on quality of life. Good history taking is necessary, with different causes to be considered: onset and duration of symptoms, previous treatments, co-existing conditions, travel and drug use may all be relevant. Tests include blood and faecal screening. Exclusion of inflammatory bowel disease and colorectal neoplasia is important and may require colonoscopy. Coeliac disease, microscopic colitis and bile acid diarrhoea are all common conditions which should not be missed, as specific therapy is available for each of these. Functional bowel disorders with diarrhoea are prevalent, overlapping with other more treatable conditions. Dietetic assessment and advice are helpful. Awareness of high FODMAP foods, with identification of individual sensitivities, is often beneficial.

\section{Introduction}

Chronic diarrhoea is a common reason for consultation in general practice and secondary care. Studies have estimated a prevalence of up to $5 \%$ in a western population. ${ }^{1}$ It can be defined as the passage of loose or watery stool (type 5-7 on the Bristol stool chart) with increased frequency, persisting for more than 4 weeks. Despite its high prevalence, making the correct diagnosis can be challenging.

First, the optimal strategy for evaluating these patients varies. Updated guidance was published from the British Society of Gastroenterology (BSG) in 2018. ${ }^{2}$ However, guidance for how, when and for how long to investigate is not the same for all patients.

Second, there is a significant overlap in symptoms between organic and functional diarrhoea, where a diagnosis is formed based on symptoms and a negative physical examination, as per the revised Rome IV criteria from 2016. 'This overlap can therefore lead to inappropriate investigations, or conversely lead to diagnostic delay, where patients are labelled as having functional diarrhoea or diarrhoea-predominant irritable bowel syndrome (IBS-D) without investigations.

Authors: ${ }^{A}$ specialist registrar, Imperial College Healthcare NHS Trust, London, UK; ${ }^{B}$ consultant gastroenterologist, Imperial College Healthcare NHS Trust, London, UK and professor, Imperial College London, London, UK
Thirdly, there are a wide range of possible causes of chronic diarrhoea that need to be considered (Table 1). It may be helpful to attempt to distinguish malabsorptive diarrhoea from inflammatory and secretory causes to help focus investigations. Particularly in malabsorption, steatorrhoea or complaints of bulky malodorous stool can be present.

The BSG guidelines provide more in-depth guidance around investigation and management of certain conditions. Here, we provide some tips to help direct investigations to make the correct diagnosis.

\section{History is key}

Every specialist says this; however, it really rings true in the approach to chronic diarrhoea. There are important discriminators that can be picked up from the history that will direct investigations for suspected diagnoses.

\section{Assess for red flag symptoms.}

The presence of red flag symptoms should prompt urgent further investigation. These symptoms include unexplained rectal

\section{Key points}

Chronic diarrhoea is a common problem with many causes in the differential diagnosis.

A thorough history is important to identify any precipitating factors.

Screening tests, including coeliac serology and faecal calprotectin, are important to diagnose common organic causes.

Bile acid diarrhoea and microscopic colitis are both common and treatable but need to be specifically looked for.

Symptoms in functional diarrhoea can be helped by attention to dietary factors such as intolerance of lactose and other FODMAPs.

KEYWORDS: functional bowel disorders, irritable bowel syndrome, bile acid diarrhoea, microscopic colitis, coeliac disease DOI: 10.7861/clinmed.2021-0028 
Table 1. Causes of chronic diarrhoea

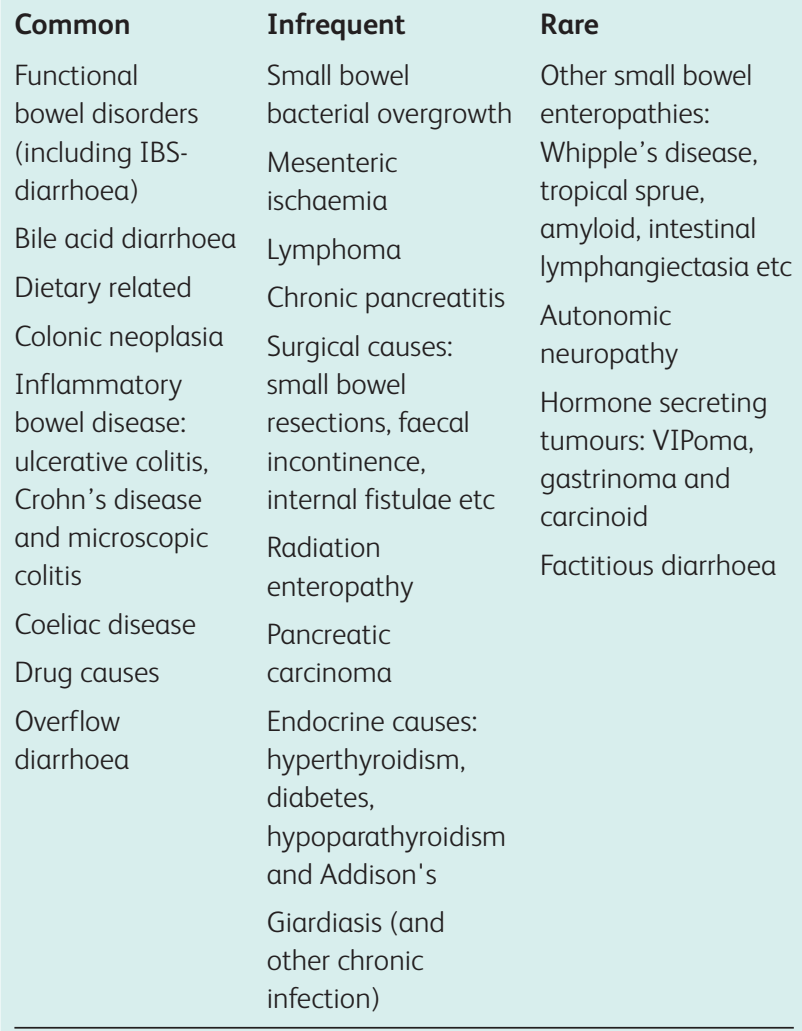

Adapted from BSG 2018. ${ }^{2}$ IBS = irritable bowel syndrome; VIPoma = vasoactive intestinal peptide tumour.

bleeding, unexplained weight loss or an unexplained change in bowel habit for more than 6 weeks in patients older than 60 years.

\section{Timing of onset}

A patient who presents with a history of diarrhoea for the past 2-3 months is more likely to have an organic pathology than a patient with diarrhoea for 3 years. Recent gastro-intestinal infections, travel, treatments (including cancer therapy) and starting new drugs are all relevant.

\section{Surgical history}

A detailed surgical history for any history of colonic resection (particularly of the right colon and terminal ileum) or cholecystectomy lead to diarrhoea due to fat and carbohydrate malabsorption and bile acid diarrhoea. Small intestinal bacterial overgrowth can also develop in these circumstances.

\section{Family history}

Asking specifically about colorectal cancer, inflammatory bowel disease (IBD) and coeliac disease in the family can help focus investigations on conditions where there may be a genetic predisposition.

\section{Common things are common}

Table 1 breaks down the various causes of chronic diarrhoea into the frequency in which they are diagnosed. All too frequently there is a delay in diagnosing common, and very treatable conditions (such as coeliac disease), with patients being labelled as having IBS-D for many years. In a 2014 population-based study of 825 people with coeliac disease, $32 \%$ reported a diagnostic delay of more than 10 years. ${ }^{3}$ Similarly, a patientorganised survey of 91 people diagnosed with bile acid diarrhoea (BAD), showed that $44 \%$ had experienced symptoms for longer than 5 years. ${ }^{4}$

To avoid missing serious or common causes for chronic diarrhoea, screening investigations should include blood tests to look for evidence of malabsorption: full blood count, renal profile, liver function tests, albumin, B12, folate, ferritin and vitamin D. Testing for $\mathrm{C}$-reactive protein has a high sensitivity for organic disease. Thyroid function tests (to exclude hyperthyroidism) and serological tests for coeliac disease (including total immunoglobulin A levels) form part of a basic screen of investigations. Faecal calprotectin is needed and can help distinguish between IBD and IBS. Values of $<50 \mu \mathrm{g} / \mathrm{g}$ make IBD unlikely, but a raised calprotectin can be found in colorectal cancer, infectious gastroenteritis and with use of non-steroidal anti-inflammatory drugs. ${ }^{5}$ Faecal immunochemical testing for haemoglobin, and faecal elastase, which is a sensitive and specific test for pancreatic exocrine insufficiency, may also be considered.

\section{Not to miss}

There can be a fine line between over- and under-investigating patients with chronic diarrhoea. Where there are red flag symptoms, the guidance for investigating is more explicit with a clear priority for excluding IBD, colonic neoplasia and causes of malabsorption. However, it can be difficult to know which patients to further investigate in secondary care to find a possible cause of chronic diarrhoea, or to simply give a diagnosis of IBS-D. When symptoms are significant enough to impact a patient's quality of life, or where simple pharmacological and lifestyle measures have not helped, further investigations are appropriate.

\section{Coeliac disease}

The prevalence of coeliac disease in the adult population in the UK is about $1 \% .{ }^{6}$ This figure may be $10 \%$ in high-risk groups with chronic diarrhoea. In light of this, coeliac disease serology with tissue transglutaminase antibodies must be checked as part of the screen of investigations in patients with chronic diarrhoea.

\section{Bile acid diarrhoea}

Bile acids are necessary for the emulsification and absorption of fats. They are absorbed in the ileum, undergoing an enterohepatic circulation to be secreted again by the liver. BAD can either result from bile acid malabsorption, usually from ileal resection or disease, or be due to overproduction of bile acids when there is impaired negative feedback regulation by FGF19. Consequently, there is excess of bile acids present in the colon, producing secretory diarrhoea. Around $30 \%$ of patients with IBS-D have BAD. The selenium homocholic acid taurine (SeHCAT) test, which measures 7-day retention of the ${ }^{75}$ Se-labelled bile acid, is recognised as the best investigation for $B A D$, and is widely available in the UK. Excessive loss of bile acids is graded as severe, moderate or mild when 7 -day retention is $<5 \%, 5-10 \%$ and $10-15 \%$, respectively, and predicts the response of these patients 
to treatment with bile acid sequestrants such as colesevelam or colestyramine. $^{7}$

\section{Microscopic colitis}

This form of IBD is less well recognised and is frequently undiagnosed. At colonoscopy the bowel is macroscopically normal, but biopsies from the left and right colon make the histological diagnosis. Excess lymphocytes in the intraepithelial and lamina propria layers, with a thickened subepithelial collagen band, give rise to the sub-types of lymphocytic and collagenous colitis, respectively. In a meta-analysis of studies of patients diagnosed with IBS-D, the prevalence of microscopic colitis was $9.8 \% .{ }^{8}$ Microscopic colitis has been linked to the use of commonly used medications (such non-steroidal anti-inflammatory drugs, proton pump inhibitors and selective serotonin reuptake inhibitors); stopping these medications may alleviate symptoms. For those that do not respond, controlled release budesonide is an effective treatment.

\section{Drugs are a frequent cause for diarrhoea}

Drug-induced diarrhoea should be considered early in the investigation of chronic diarrhoea and is an important part of the initial history. ${ }^{9}$ More than 700 medications implicate diarrhoea as an adverse reaction and, in an ageing population with complex comorbidities, we are likely to encounter drug-induced diarrhoea with increasing frequency. Multiple overlapping mechanisms can lead to drug-induced diarrhoea and these include osmotically active substances drawing water into the bowel, inhibition of electrolyte and nutrient absorption or by increasing the secretion of electrolytes into the intestinal lumen, prokinetic effects on the lumen, or causing epithelial inflammation. Commonly implicated medications include antibiotics (particularly macrolides like erythromycin), nonsteroidal anti-inflammatory drugs, magnesium-containing products, hypoglycaemic agents (especially metformin and dipeptidyl peptidase- 4 inhibitors), antineoplastic agents, and cardiovascular medications (furosemide and angiotensinconverting enzyme inhibitors). Where possible, withdrawal of the offending medications is suggested; however, this will need to be done on a risk/benefit basis.

\section{Dietary tips in chronic diarrhoea}

A patient's diet can play a part both in causing and exacerbating symptoms in chronic diarrhoea. There is no 'one size fits all approach', and this can lead to confusing and contradictory patient advice. A 'healthy diet', which is high in fruits and vegetables, and contains proteins, carbohydrates and fats may lead to worsening of their symptoms. Particular forms of carbohydrate that can trigger digestive symptoms are the fermentable oligo-, di-, mono-saccharides and polyols (FODMAPs), shown in Table 2. These are poorly digested and poorly absorbed, consequently reaching the colon where they are fermented by bacteria. This can lead to diarrhoea, bloating and abdominal pain. Meta-analyses have shown improvement in patient symptoms and quality of life by reducing FODMAP intake. ${ }^{10}$

Lactose malabsorption is an example of FODMAP sensitivity. This occurs as a result of lactase non-persistence, the usual adult human global phenotype, due to down-regulation of the enzyme
Table 2. Some foods that are high in FODMAPS

(fermentable oligo-, di-, mono-saccharides and polyols)

$\begin{array}{ll}\text { Fructose } & \text { Apples, mangos, pears and fruit juices } \\ \text { Lactose } & \text { Milk and unripened cheeses } \\ \text { Fructans } & \text { Onions, garlic, cabbage, broccoli and cereals } \\ \text { Galactans } & \text { Beans, lentils and chickpeas } \\ \text { Polyols } & \text { Mushrooms, nectarines and avocados }\end{array}$

after weaning in childhood. Evolution has developed lactase persistence in certain populations, for example in north-west Europe, but it remains a common cause of diarrhoea. A trial of dairy exclusion from the diet may show an improvement in symptoms. A hydrogen breath test can give a definite diagnosis where there is doubt.

Another FODMAP source are the polyols (sorbitol and xylitol), artificial sweeteners commonly found in chewing gum and soft drinks, which can also exacerbate symptoms of bloating and diarrhoea.

\section{References}

1 Lacy BE, Mearin F, Chang L et al. Bowel Disorders. Gastroenterology 2016;150:1393-407.

2 Arasaradnam RP, Brown S, Forbes A et al. Guidelines for the investigation of chronic diarrhoea in adults: British Society of Gastroenterology, 3rd edition. Gut 2018:67:1380-99.

3 Fuchs V, Kurppa K, Huhtala $\mathrm{H}$ et al. Factors associated with long diagnostic delay in celiac disease. Scand J Gastroenterol 2014;49:1304-10.

4 Bannaga A, Kelman L, O'Connor M et al. How bad is bile acid diarrhoea: an online survey of patient-reported symptoms and outcomes. BMJ Open Gastroenterol 2017;4:e000116.

5 Waugh N, Cummins E, Royle P et al. Faecal calprotectin testing for differentiating amongst inflammatory and non-inflammatory bowel diseases: systematic review and economic evaluation. Health Technol Assess 2013;17:xv-xix, 1-211.

6 Hopper AD, Cross SS, Hurlstone DP et al. Pre-endoscopy serological testing for coeliac disease: evaluation of a clinical decision tool. BMJ 2007:334:729.

7 Walters JRF, Arasaradnam RP, Andreyev HJN. Diagnosis and management of bile acid diarrhoea: a survey of UK expert opinion and practice. Frontline Gastroenterol 2019;11:358-63.

8 Guagnozzi D, Arias A, Lucendo AJ. Systematic review with metaanalysis: diagnostic overlap of microscopic colitis and functional bowel disorders. Aliment Pharmacol Ther 2016:43:851-62.

9 Philip NA, Ahmed N, Pitchumoni CS. Spectrum of drug-induced chronic diarrhea. J Clin Gastroenterol 2017;51:111-7.

10 Marsh A, Eslick EM, Eslick GD. Does a diet low in FODMAPs reduce symptoms associated with functional gastrointestinal disorders? A comprehensive systematic review and meta-analysis. Eur ] Nutr 2016:55:897-906

Address for correspondence: Prof Julian Walters, Gastroenterology Department, Hammersmith Hospital, Du Cane Road, London W12 0HS, UK. Email: julian.walters@imperial.ac.uk Twitter: @jrfw51 